\section{ORIGINAL RESEARCH}

\author{
P.G. Kranz \\ L. Gray \\ J.N. Taylor
}

\title{
CT-Guided Epidural Blood Patching of Directly Observed or Potential Leak Sites for the Targeted Treatment of Spontaneous Intracranial Hypotension
}

BACKGROUND AND PURPOSE: Optimal diagnosis and management of spontaneous intracranial hypotension remains uncertain. CT-guided blood patching has been described but has not been evaluated in larger case series. We sought to evaluate the efficacy of CT-guided blood patching of observed or potential CSF leaks in spontaneous intracranial hypotension.

\begin{abstract}
MATERIALS AND METHODS: Patients referred for evaluation of spontaneous intracranial hypotension were retrospectively reviewed. Inclusion criteria were findings of intracranial hypotension on pretreatment brain MR imaging, evaluation and treatment with CT-guided myelography and blood patching, and availability of posttreatment brain MR images. Eight patients met inclusion criteria. Imaging findings, treatment details, and clinical outcomes were assessed.
\end{abstract}

RESULTS: Pretreatment imaging findings included dural enhancement, tonsillar ectopia, subdural collections, and syrinx. All findings resolved or significantly improved on posttreatment imaging. Presenting clinical symptoms included positional headache, neck/interscapular/shoulder pain, and tinnitus. Headaches and neck/interscapular/shoulder pain improved in all patients; tinnitus improved in 1 of 2 patients. CSF leak sites were directly visualized in $37 \%$ of patients and were targets for patching when seen. When no direct visualization of leaks was seen, irregular spinal nerve root diverticula were targeted as potential leak sites. The average number of blood patching sessions was 3 (range, 1-6) and the average number of individual sites patched per session was 5 (range, 1-10).

CoNCLUSIONS: Our results suggest that CT-guided blood patching targeting observed or potential leak sites can be effective in the treatment of intracranial hypotension. Prospective controlled studies are needed to confirm efficacy and compare outcomes with other treatment options.

ABBREVIATIONS: NA $=$ date not available

$S_{\text {diag }}$ pontaneous intracranial hypotension is a challenge both diagnostically and therapeutically due to the varied clinical and imaging findings on presentation, incomplete understanding of the underlying pathophysiology, and uncertainty about optimal management. A variety of therapeutic options, including conservative therapy, surgery, and nontargeted and image-guided epidural blood patching, have been described. There remains disagreement, however, about the effectiveness and rationale for selection of the various modalities. CTguided blood patching has been described as a treatment option for intracranial hypotension, but information has been limited to case reports, with no larger case series available. Image-guided blood patching has also previously relied on direct visualization of leak sites. Although in some cases a single or multiple leak sites can be directly observed, in many patients, the precise site of CSF extravasation is not identified

Received June 25, 2010; accepted after revision September 19.

From the Department of Radiology, Duke University Medical Center, Durham, North Carolina.

Previously presented at: Annual Meeting of the American Society of Neuroradiology, May 15-20, 2010; Boston, Massachusetts.

Please address correspondence to Peter G. Kranz, MD, Department of Radiology, Box 3808, Duke University Medical Center, Durham, NC 27710; e-mail: peter.kranz@duke.edu

Indicates open access to non-subscribers at www.ajnr.org

Indicates article with supplemental on-line tables.

DOI 10.3174/ajnr.A2384 on imaging studies. We sought to determine whether implementation of CT-guided blood patching targeting potential or observed leakage sites involving the spinal nerve roots could result in clinical remission of symptoms and reversal of imaging findings.

\section{Materials and Methods}

This investigation is a retrospective case series of patients referred for the treatment of clinically suspected intracranial hypotension between February 2005 and June 2009. The study was performed with approval from our local institutional review board. Inclusion criteria were the following: presence of imaging signs of intracranial hypotension on pretreatment brain MR imaging, concurrent diagnosis and treatment with CT-guided myelography and blood patching, and availability of posttreatment brain MR imaging.

Evaluation with CT-guided myelography followed by immediate treatment with CT-guided blood patching was performed on an outpatient basis in all patients. In this procedure, patients are placed in the left lateral decubitus position on the CT gantry, and CT fluoroscopic guidance is used to perform a lumbar puncture with a 22 -ga spinal needle. Opening pressure is measured with the patient resting quietly and with legs extended. Ten milliliters of contrast (iopamidol, Isovue-M 300; Bracco Diagnostics, Princeton, New Jersey) is instilled into the thecal sac, and the intrathecal location is confirmed with CT fluoroscopy. The needle is removed, and the patient is returned to the supine position. The patient is assisted in performing a pelvic lift on the gantry table by bracing his or her feet against a bolster and lifting 

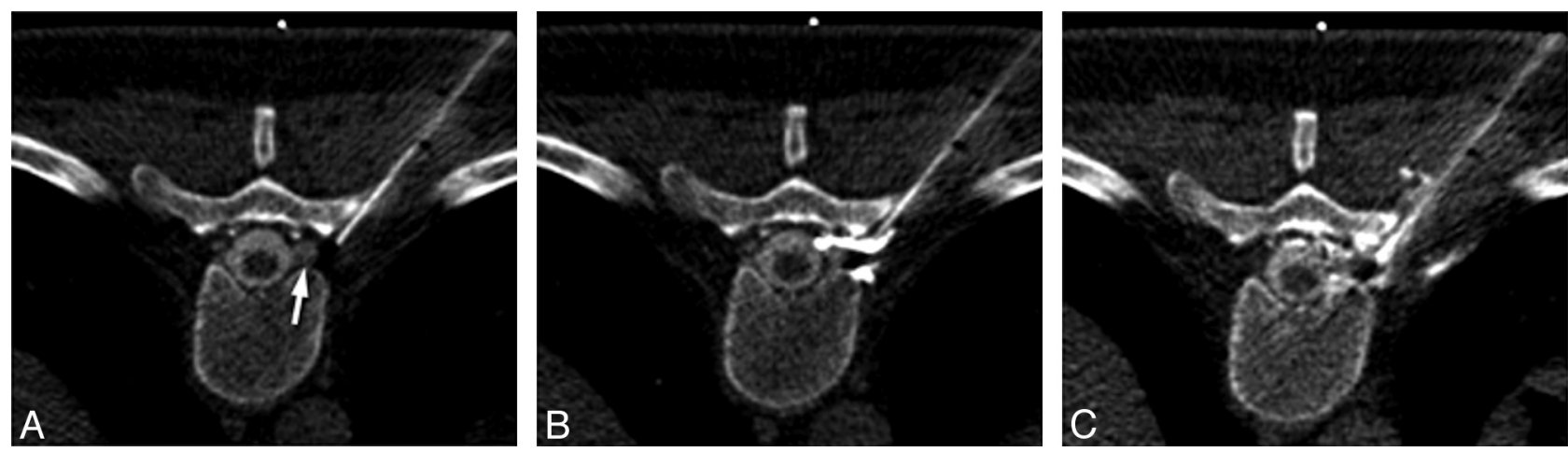

Fig 1. Technique for CT-guided transforaminal blood patching. $A$, Needle placement before patching. The needle tip is located in the posterior third of the neural foramen, adjacent to

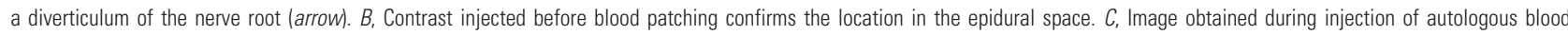
demonstrates displacement of the contrast by the injected blood and mild mass effect on the diverticulum.

the pelvis off the gantry with assistance from the operators, a maneuver that allows flow of contrast into the thoracic and cervical spine. The patient is rolled $540^{\circ}$ to further distribute the contrast and then is placed prone. CT scanning of the cervical, thoracic, and lumbar spine from the skull base to the sacrum is performed. The images are immediately evaluated at the scanner for evidence of frank leakage of contrast into the epidural space or irregular diverticula of the nerve roots that may serve as potential leak sites. Observed leak sites are patched when visualized; when no direct site of leakage is seen, potential leak sites are targeted for patching under CT fluoroscopic guidance.

The blood patches are planned, and the skin is marked for the CT-guided procedure, which is performed concurrently with the CT myelography. A 22-ga spinal needle is inserted at the marked site and directed to the location for the blood patching via a transforaminal epidural approach. Intermittent CT fluoroscopy and a low milliampere technique are used to minimize the overall radiation dose. Care is taken not to advance the needle beyond the posterior third of the neural foramen to minimize the risk of injury to adjacent vessels or nerves. The epidural location of the needle adjacent to the nerve root and the flow of contrast into the neural foramen are confirmed with approximately $0.2 \mathrm{~mL}$ of contrast (Isovue-M 200; Bracco Diagnostics). The contrast injection is followed by an injection of approximately $3 \mathrm{~mL}$ of autologous blood (Fig 1). A slightly lower volume of blood $(1.5-2 \mathrm{~mL})$ is occasionally needed depending on mass effect from the blood patch. Following the procedure, patients are typically contacted by telephone for follow-up several times in the first month and again at approximately $6-9$ months, unless recurrent symptoms necessitate earlier follow-up. Patients underwent repeated procedures if they experienced only partial relief or had recurrent symptoms following an initial response to blood patching.

Imaging studies were retrospectively reviewed for identified patients who underwent CT-guided myelography and blood patching. Patients were considered to have imaging-confirmed intracranial hypotension if pretreatment MR imaging demonstrated smooth dural thickening and enhancement or $\geq 2$ of the following: subdural collections, marked narrowing of the suprasellar and/or perimesencephalic cisterns, or cerebellar tonsillar ectopia. If cerebellar tonsillar ectopia was the only MR imaging finding, the imaging was considered to be equivocal. Posttreatment imaging was considered to demonstrate resolution of findings if there was normalization of dural enhancement (if it was abnormal on preprocedural imaging) and marked improvement or resolution of other associated imaging findings of intracranial hypotension.
Imaging findings on pre- and posttreatment MR imaging, findings of CT myelography, CT fluoroscopy time from the initial blood patching procedure, type and number of leaks or potential leaks treated with blood patching, and total number of treatment sessions between pretreatment and posttreatment MR imaging were recorded. Presenting clinical symptoms and follow-up symptoms were also recorded. Time intervals between pretreatment MR imaging and blood patching and the interval from blood patching to posttreatment MR imaging were recorded. If $>1$ session of blood patching was performed due to ongoing clinical symptoms, the myelographic findings and number of patches were determined from the initial session and the time interval to posttreatment MR imaging was based on the time from the most recent blood patch. The Spearman rank correlation coefficient was used to assess the relationship between the number of sites treated at the initial blood patching session and the number of return sessions the patient required.

\section{Results}

Twenty-one patients were identified who were referred for evaluation for clinically suspected intracranial hypotension who also had positive findings on pretreatment brain MR imaging. Imaging findings were considered to be definitively positive in 15 cases and equivocal (demonstrating only cerebellar tonsillar ectopia) in 6 cases. Of the cases considered to be definitively positive, 8 patients had follow-up imaging.

Demographic information, symptoms, and clinical outcomes for these 8 patients are presented in On-line Table 1. Seven patients $(88 \%)$ had headaches at the time of presentation, 6 of whom had a positional component to them. The patient without headaches at presentation had chest and arm pain with lower extremity weakness (patient 4) but did have a longstanding history of headaches. Six patients (75\%) had neck, interscapular, or shoulder pain. Two patients (25\%) had tinnitus. Following treatment, all patients had improvement in their positional headaches, though 1 patient later re-presented with nonpositional headaches due to posttreatment increased CSF pressure. All patients with pretreatment neck, interscapular, or back pain reported significant improvement. Of the patients with tinnitus, 1 had resolution, while the other had symptoms that persisted after resolution of her headaches.

MR imaging findings, procedural details, and time intervals between pretreatment imaging, blood patching, and follow-up imaging are presented in On-line Table 2. Dural enhancement was the most common MR imaging finding, 


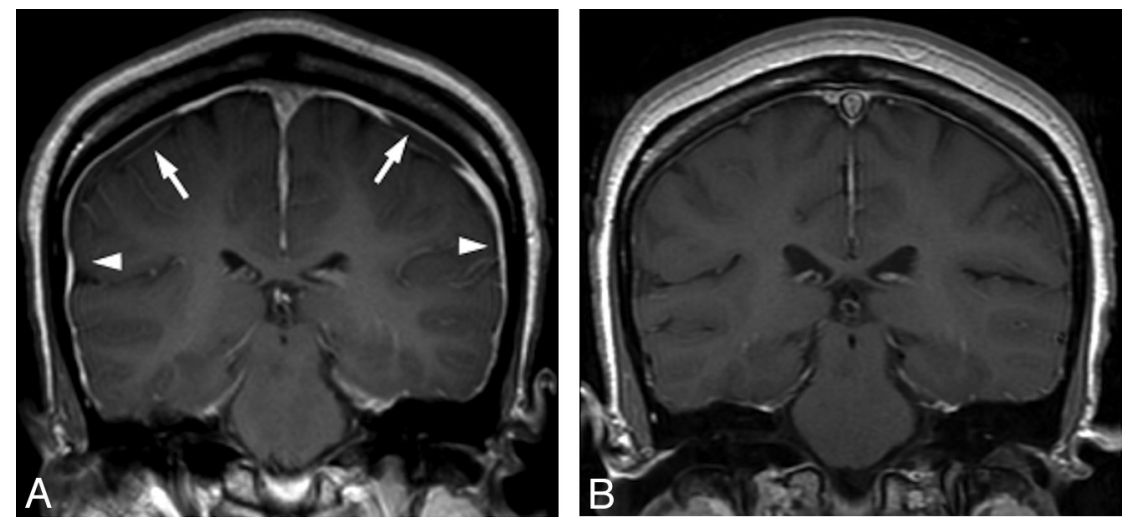

Fig 2. Resolution of imaging changes of intracranial hypotension. $A$, Pretreatment MR image shows typical signs of intracranial hypotension, including smooth dural thickening and enhancement (arrowheads) and subdural collections (arrows). B, Follow-up brain MR image after CT-guided targeted blood patching demonstrates resolution of dural enhancement and subdural collections
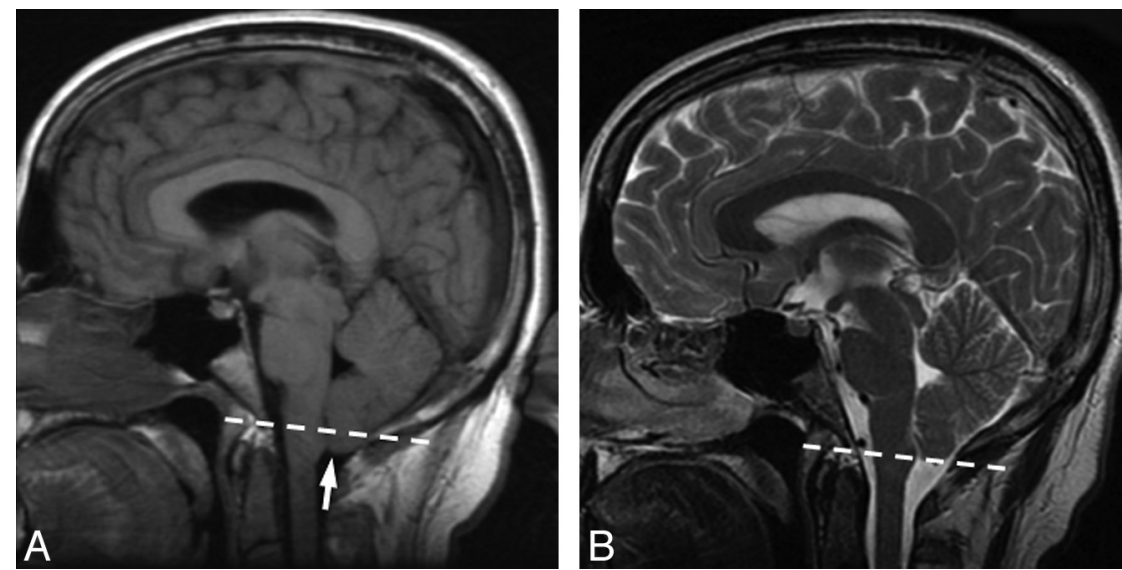

Fig 3. Pre- and posttreatment MR imaging from patient 2. Dashed line indicates the foramen magnum. A, Pretreatment MR image demonstrates cerebellar tonsillar ectopia (arrow). The dashed line indicates the plane of the foramen magnum. Midline sagging of the brain with inferior displacement of the mammillary bodies is seen. In this patient, there was no abnormal dural enhancement on pretreatment MR imaging (not shown). $B$, Follow-up brain MR image after CT-guided blood patching demonstrates resolution of the cerebellar tonsillar ectopia and midline sag.

present in 7 (88\%) patients. In 3 of these patients, this was the only imaging finding. Cerebellar tonsillar ectopia was observed in $5(63 \%)$ patients and was observed with concomitant dural enhancement in all except 1 of the cases considered to be definitively positive on imaging. Intracranial subdural collections were seen in $2(25 \%)$ patients.

In all patients, these findings resolved or significantly improved following blood patching. Dural enhancement resolved in all patients in whom this was seen on pretreatment imaging (Fig 2). In the single patient without dural enhancement, both the cerebellar tonsillar ectopia and midline sag resolved on posttreatment imaging (Fig 3). In 1 patient, the cerebellar tonsillar ectopia resulted in cervical and thoracic syringohydromyelia at the time of presentation (patient 4). Following blood patching in this patient, there was resolution of the tonsillar ectopia and near-complete resolution of the syringohydromyelia.

Patients had been symptomatic for an average of 11.5 months before evaluation (range, 18 days to 24 months). Three of the 8 patients had previously undergone multiple ( 2 or 3 ) nontargeted lumbar epidural blood patches without resolution of their symptoms. The median time between pretreatment MR imaging and treatment was 19 days (range,
4-140 days), and the median time from blood patching to posttreatment follow-up was 48 days (range, 7-490 days).

Findings of CT-myelography are displayed in the Table. Irregular thoracic meningeal diverticula were identified in 7 $(88 \%)$ patients. Coexistent large spinal CSF collections were observed in 3 (37\%) patients, 2 of which were extradural and the other subdural. The single patient without irregular diverticula identified on myelography (patient 3) had 1 of these extradural fluid collections, which extended along adjacent nerve root sleeves. On follow-up images, these collections significantly decreased in size (Fig 4). The average number of CT-targeted blood patches (ie, individual sites treated) performed at the initial treatment session was 5 (range, 1-10). Total CT fluoroscopy time from the initial procedure averaged 45 seconds (range, 18-72 seconds). Average CT fluoroscopic time per site treated on the initial procedure was 10 seconds. The average number of blood-patching sessions between the initial pretreatment MR imaging and the posttreatment MR imaging was 3 (range, 1-6). There was no correlation between the number of sites treated on the initial visit and the total number of treatment sessions required (Spearman $r=0$ ). No immediate or delayed procedural complications were observed. 


\begin{tabular}{|c|c|c|c|c|c|c|c|c|}
\hline \multicolumn{9}{|c|}{ Findings of CT myelography ${ }^{a}$} \\
\hline \multirow[b]{2}{*}{$\begin{array}{l}\text { Patient } \\
\text { No. }\end{array}$} & \multirow[b]{2}{*}{$\begin{array}{l}\text { Opening Pressure } \\
\left(\mathrm{cm} \mathrm{H}_{2} \mathrm{O}\right)\end{array}$} & \multirow[b]{2}{*}{$\begin{array}{l}\text { Spinal Fluid } \\
\text { Collection }\end{array}$} & \multirow[b]{2}{*}{$\begin{array}{l}\text { Irregular } \\
\text { Diverticula }\end{array}$} & \multirow[b]{2}{*}{$\begin{array}{l}\text { Direct Visualization } \\
\text { of Leak }\end{array}$} & \multicolumn{4}{|c|}{ Location of Diverticula } \\
\hline & & & & & Cervical & $\begin{array}{c}\text { Upper } \\
\text { Thoracic } \\
(\mathrm{T} 1-6)\end{array}$ & $\begin{array}{c}\text { Lower } \\
\text { Thoracic } \\
\text { (T6-12) }\end{array}$ & Lumbar \\
\hline 1 & 0 & + & + & + & 0 & 4 & 0 & 0 \\
\hline 2 & 15 & - & + & - & 0 & 1 & 2 & 0 \\
\hline 3 & 0 & + & - & + & 0 & 5 & 0 & 0 \\
\hline 4 & $\mathrm{~N} / \mathrm{A}$ & - & + & - & 0 & 0 & 7 & 0 \\
\hline 5 & $\mathrm{~N} / \mathrm{A}$ & - & + & - & 0 & 0 & 2 & 0 \\
\hline 6 & 0 & + & + & + & 0 & 0 & 1 & 0 \\
\hline 7 & 0 & - & + & - & 0 & 2 & 5 & 1 \\
\hline 8 & 12 & - & + & - & 0 & 1 & 4 & 5 \\
\hline
\end{tabular}

Note: ${ }^{a}+$ indicates presence of the finding; - , absence of the finding.

\section{Discussion}

Our investigation found that all patients with available preand posttreatment imaging who underwent CT-guided blood patching demonstrated both subjective improvement in symptoms and objective reversal of imaging findings of intracranial hypotension. These findings are important in that they suggest that in patients presenting with intracranial hypotension, implementation of CT-guided blood patching targeted at potential or observed leak sites can reverse the abnormal physiologic changes seen on imaging and effectively treat symptoms. Changes in imaging findings included not only reversal of dural enhancement but also resolution of intracranial subdural collections, resolution or near-resolution of spinal fluid collections, reversal of cerebellar tonsillar ectopia, and in 1 case, reversal of syringohydromyelia. CT-guided blood patches were effective at treating not only positional headaches but also other associated symptoms, including neck and back pain and tinnitus in some cases.

Optimal treatment strategies for spontaneous intracranial hypotension have not yet been clearly established. We undertook this retrospective study to determine if the targeting of multiple observed or potential leak sites with CT-guided blood patching might provide an alternative strategy for treatment that was minimally invasive and directed at the suspected underlying anatomic pathology. While previous series have examined conservative therapy, surgical therapy, and epidural blood patching without the guidance of cross-sectional imaging, reports of CT-guided blood patching have predominantly consisted of single case reports. ${ }^{1-3}$ Conservative management of intracranial hypotension consists of bed rest, hydration, and caffeine for symptomatic relief. Although effective for some patients, recovery times can be lengthy and patients may be left with residual headache symptoms. ${ }^{4}$ Surgical ligation of leaking nerve root sleeves has been reported but requires confident preoperative localization of the leak site, is relatively invasive, and has limited ability to address multiple potential leak sites. ${ }^{5}$

Large-volume lumbar epidural blood patches, an approach adapted from the treatment of post-lumbar puncture headache, are often used for treatment of spontaneous intracranial hypotension and can be effective immediately. ${ }^{6}$ However, it remains unclear how such therapy might actually address the underlying problem of spontaneous CSF leaks, given that when visualized, these leaks are most commonly observed in the thoracic region, not the lumbar region. ${ }^{7}$ One possibility is that these nondirected "patches" increase CSF pressure by displacement of volume in the spinal canal, thereby alleviating symptoms until spontaneous closure of leaks can occur. Although this technique can be effective for some patients, it is not as effective as epidural blood patching performed in the setting of post-lumbar puncture headaches, is generally less effective when the patch is placed distant from an identified leak site, and may not achieve durable results in a significant proportion of patients. ${ }^{8,9}$ Reports of the overall rates of success for lumbar epidural blood patching vary significantly. While some series report success rates in excess of $80 \%$, several studies have found success rates to be substantially lower, and several authors have reported success rates of $<50 \% .^{8,10-15}$ In our series, 3 of 8 patients had failed multiple prior nontargeted lumbar epidural blood patches. Alternative treatment strategies for such patients are, therefore, important.

In cases of spontaneous intracranial hypotension, the observation of large or irregular diverticula associated with the spinal nerve roots and occasional instances of frank extravasation of intrathecally injected contrast or radionuclide from nerve root sheaths on imaging studies has implicated the nerve root sheath as a weak spot that may be prone to spontaneous rupture. ${ }^{16,17}$ Most patients in our series demonstrated irregular diverticula associated with spinal nerve root sleeves, and these were selected as the treatment sites. The decision to treat these sites with blood patches was empiric, on the basis of their potential as leak sites that could be intermittent or low-flow, recognizing that not all the patches placed would cover sites of active leakage. In a few cases of patients with high-flow leaks, however, actual extravasation of contrast was observed at myelography (Fig 5).

The exact pathogenesis of diverticula of nerve root sleeves and under what circumstances these lead to leaks is unknown. It has been suggested that these diverticula may form as the result of an underlying connective tissue disorder. ${ }^{18}$ An alternative possibility we suggest is that the diverticula may in some cases represent abnormally enlarged arachnoid granulations. The spinal nerve roots are known to be normal sites of egress of CSF from the subarachnoid space back into the lymphatic system; Brierley ${ }^{19}$ demonstrated that ink injected into the ventricular system of rabbits could be found in the subarachnoid space of the nerve roots, as well as in the lumbosacral and cervical paraspinal lymph nodes. In humans, penetration of arachnoid granulations through the dura of the spinal nerve root sheath into adjacent radicular veins allows resorption of 

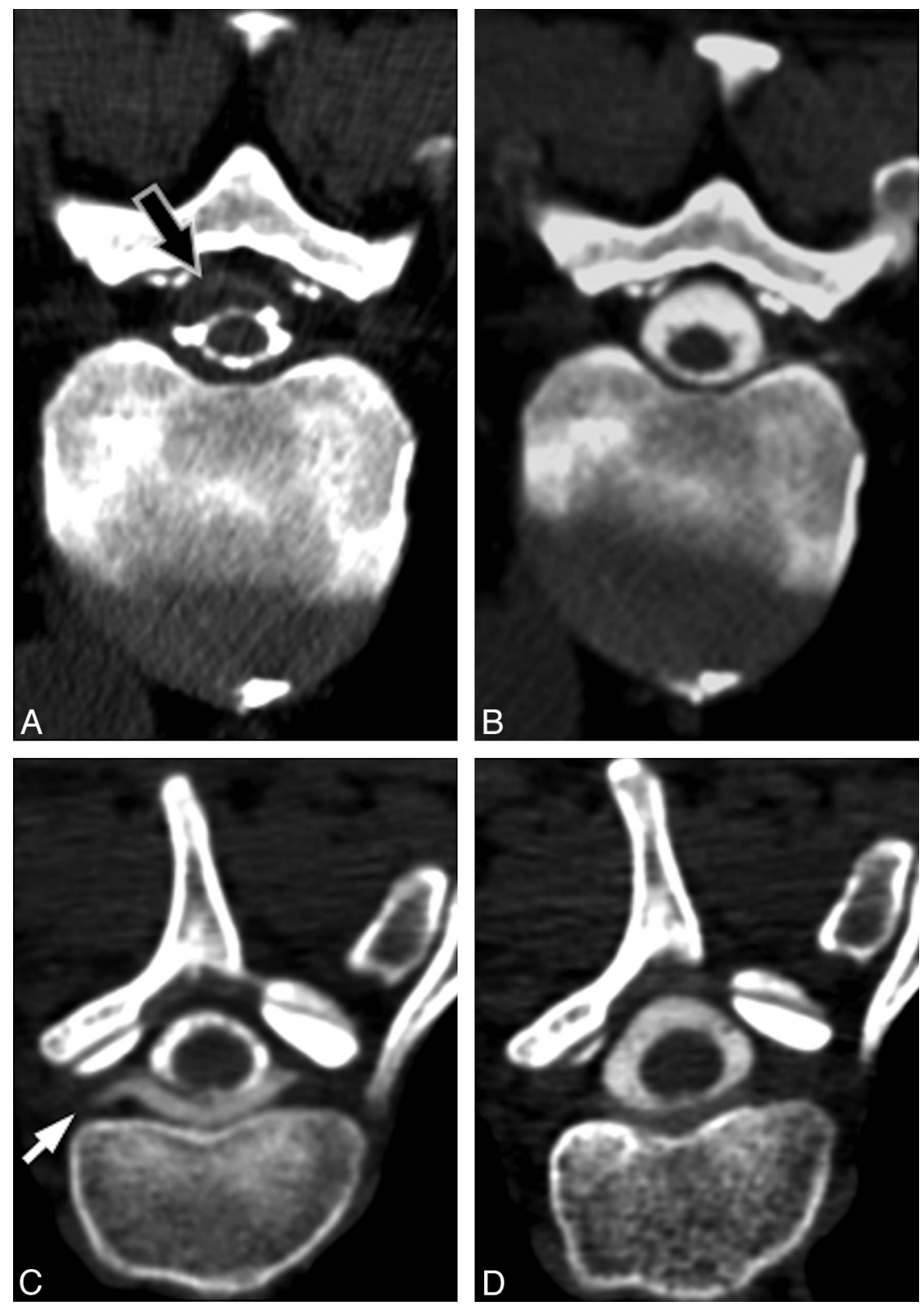

Fig 4. Pre- and posttreatment appearance of spinal fluid collections. A, Pretreatment CT myelogram from patient 6 demonstrates a subdural fluid collection (arrow) in the spinal canal, which narrows the CSF space. B, Follow-up CT myelogram 15 days after CT-guided blood patching at the site of leak demonstrates resolution of the subdural collection. $C$, Pretreatment CT myelogram from patient 3 demonstrates an extradural collection ventral to the thecal sac, extending into the nerve root sleeves (arrow). D, Follow-up CT myelogram 17 days after CT-guided blood patching of the nerve root sleeves demonstrates near-complete resolution of the collection.

CSF; however, the degree to which such penetrating arachnoid granulations occur varies from person to person and may result in individual differences in CSF egress via this route. ${ }^{20}$ Regardless of their precise etiology, our data support targeting these diverticula as sites for blood patching. Further investigation is needed for predicting how to distinguish potential from actual leak sites in patients with multiple diverticula and the frequency with which such diverticula may be seen in asymptomatic patients on CT myelography.

Typically, patients with intracranial hypotension have positional headaches that are relieved in the recumbent position and exacerbated with standing. ${ }^{21}$ A variety of other less common symptoms may be present, however, including nonpositional chronic headaches, migraine-type headaches, neck and upper back pain, cranial neuropathies, and endocrine or au- tonomic dysfunction. ${ }^{22}$ Often, clinical presentations may be quite varied; this variation has led to efforts to redefine the diagnostic criteria by using a combination of clinical symptoms, imaging findings, and CSF pressures. ${ }^{23}$ All patients in our case series satisfied these proposed revised diagnostic criteria. In our series, concurrent neck, back, or interscapular pain was present in most patients, possibly caused by traction on cervical nerve roots due to downward displacement of the brain and spinal cord. One patient presented with chest and arm pain and lower extremity weakness due to tonsillar herniation and syringohydromyelia associated with clear imaging evidence of intracranial hypotension but without positional headache. Awareness of these less typical manifestations of intracranial hypotension may help avoid mistaken attribution of such symptoms to musculoskeletal or other etiologies. ${ }^{24}$ 


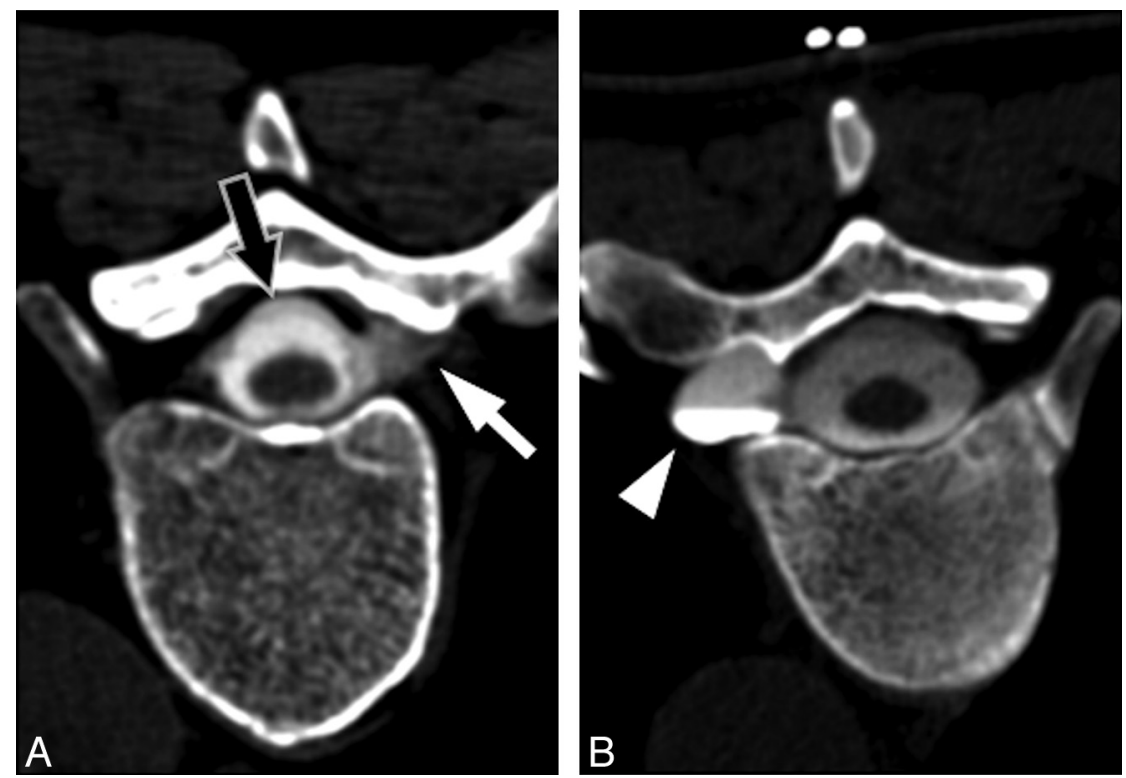

Fig 5. Representative examples of targets for blood patching. A, CT myelogram demonstrates extravasation of contrast from a nerve root sleeve (white arrow) due to a high-flow leak. Note the presence of an extradural collection surrounding the thecal sac (black arrow). B, CT myelogram in a different patient demonstrates an enlarged diverticulum of the nerve root sleeve (arrowhead). Although no direct myelographic evidence of leak is seen, this site was chosen for empiric blood patching on the basis of the potential for an intermittent or low-flow leak.

Imaging evidence of intracranial hypotension usually, but not always, includes dural enhancement. The absence of enhancement should not exclude the diagnosis. ${ }^{25}$ In the single patient in our series without evidence of dural enhancement on the pretreatment imaging, posttreatment imaging clearly demonstrated interval change in cerebellar tonsillar ectopia (Fig 3). We interpret this to represent physiologic alterations in CSF dynamics leading to restoration of buoyancy to the intracranial compartment as a result of arrested CSF leakage. Interestingly, this patient returned with headaches after blood patching and was found to have intracranial hypertension with an opening pressure of $32 \mathrm{~cm} \mathrm{H}_{2} \mathrm{O}$, and responded immediately to removal of $20 \mathrm{~mL}$ CSF. This "rebound" increased pressure may be due to temporary displacement of CSF volume associated with blood patching, a phenomenon we have increasingly recognized in our practice, which can be managed with posttreatment acetazolamide until resolution of symptoms. ${ }^{26}$

Spinal fluid collections are also commonly identified in cases of spontaneous intracranial hypotension. ${ }^{27}$ In our series, 2 extradural collections and 1 subdural collection were identified. Although less common than extradural collections in our experience, subdural collections have been reported previously. ${ }^{28,29}$ In our case, targeted blood patching in the epidural space adjacent to the site of the visualized leak resulted in resolution of the collection, presumably due to mass effect, which compressed the dura against the arachnoid, thereby preventing further leakage.

As the total number of sites treated with CT-guided blood patching increases, the total volume of blood injected into the epidural space increases. One might speculate that the volume of blood injected might lead to a temporary decrease in symptoms due to simple displacement of CSF, similar to the proposed mechanism for nontargeted lumbar blood patches. Although the size of our study was small, we found no correlation between the number of sites patched at the initial treatment (and thus the total volume of blood injected) and the number of return sessions. Furthermore, resolution of symptoms and reversal of imaging findings were seen with as little as $3 \mathrm{~mL}$ of total blood volume injected. This suggests that there is a mechanism other than simple displacement of volume that accounts for abatement of symptoms and observed imaging changes. We hypothesize that this mechanism is direct sealing of CSF leaks from their site of origin and/or decreased egress of CSF from these nerve root diverticula.

The retrospective nature of this study presents several limitations. A relatively small number of patients was included in this case series, in part due to our requirement for posttreatment imaging. The absence of a control group precludes an assessment of the efficacy of CT-guided blood patching compared with other treatments. As mentioned above, comparative efficacy data for the treatment of spontaneous intracranial hypotension in general are severely lacking. In this study, however, the average duration of symptoms at the time of initial blood patching was $>11$ months, suggesting that continuing conservative therapy alone would have likely been ineffective. Moreover, we believe that the duration of symptoms before treatment makes less likely (though by no means eliminates) the possibility that the improvement observed represents regression toward the mean. Prospective controlled studies are needed to confirm the benefit we observed and to compare nondirected lumbar epidural blood patching with CT-guided blood patching.

An additional limitation of this study was the lack of uniformity in time to follow-up imaging. Although most patients in this series had follow-up imaging within 2 months, there were a few outliers for whom the time to follow-up imaging was substantially longer. Because follow-up imaging is not part of routine care, the time intervals to repeat imaging can be expected to vary significantly. However, the patients with the 
longest times to follow-up imaging had generally been symptomatic for greater periods of time before therapy. Furthermore, improvement in clinical symptoms preceded the imaging re-evaluation. We think that it is unlikely, therefore, that there would have been spontaneous resolution of symptoms unrelated to blood patching in the time interval between therapy and follow-up imaging. Moreover, these extended follow-up times demonstrate the durability of the treatment for some patients. Because we selected only patients with follow-up imaging, there is the potential for unrecognized selection bias, which again will be best mitigated with future prospective studies. Future studies may also benefit from standard imaging follow-up times to assess the expected time course for physiologic changes associated with successful treatment.

Radiation dose is a consideration when performing CTguided blood patching. Radiation exposure comes from both the diagnostic CT myelogram and the therapeutic CT-fluoroscopically guided blood patching. Although MR myelography is performed at some institutions for diagnostic purposes, the safety of intrathecal gadolinium remains uncertain, and the sensitivity of this technique compared with CT myelography has not been established, to our knowledge. Relative to the diagnostic portion of the examination, the dose from the CTfluoroscopically guided blood patching is small. In our series, use of intermittent fluoroscopy averaging 10 seconds of fluoroscopic time per site patched, combined with reducing the milliamperes for the therapeutic portions of the procedure, helps to decrease the overall dose. Nevertheless, the radiation dose should be considered when comparing the risks and benefits of different therapeutic modalities.

\section{Conclusions}

In summary, our data suggest that CT-guided blood patching can be an effective treatment for spontaneous intracranial hypotension, as demonstrated by both clinical and imaging response. This therapy offers a more targeted and minimally invasive approach to treating the presumed etiology, based on current understanding of the disease process. Further controlled studies are needed to address comparative efficacy among treatment modalities, including the short- and longterm risk of symptom recurrence.

\section{References}

1. Karst M, Hollenhorst J, Fink M, et al. Computerized tomography-guided epidural blood patch in the treatment of spontaneous low cerebrospinal fluid pressure headache. Acta Anaesthesiol Scand 2001;45:649-51

2. Rai A, Rosen C, Carpenter J, et al. Epidural blood patch at C2: diagnosis and treatment of spontaneous intracranial hypotension. AJNR Am J Neuroradiol 2005;26:2663-66

3. Gladstone JP, Nelson K, Patel N, et al. Spontaneous CSF leak treated with percutaneous CT-guided fibrin glue. Neurology 2005;64:1818-19
4. Kong DS, Park K, Nam DH, et al. Clinical features and long-term results of spontaneous intracranial hypotension. Neurosurgery 2005;57:91-96; discussion 91-96

5. Schievink WI, Morreale VM, Atkinson JL, et al. Surgical treatment of spontaneous spinal cerebrospinal fluid leaks. J Neurosurg 1998;88:243-46

6. Berroir S, Loisel B, Ducros A, et al. Early epidural blood patch in spontaneous intracranial hypotension. Neurology 2004;63:1950-51

7. Inamasu J, Guiot BH. Intracranial hypotension with spinal pathology. Spine J 2006;6:591-99

8. Sencakova D, Mokri B, McClelland RL. The efficacy of epidural blood patch in spontaneous CSF leaks. Neurology 2001;57:1921-23

9. Duffy PJ, Crosby ET. The epidural blood patch: resolving the controversies. Can J Anaesth 1999;46:878-86

10. Horikoshi T, Watanabe A, Uchida M, et al. Effectiveness of an epidural blood patch for patients with intracranial hypotension syndrome and persistent spinal epidural fluid collection after treatment. J Neurosurg 2010;113:940-6. Epub 2009 Nov 13

11. Franzini A, Messina G, Nazzi V, et al. Spontaneous intracranial hypotension syndrome: a novel speculative physiopathological hypothesis and a novel patch method in a series of 28 consecutive patients. I Neurosurg 2010;112:300-06

12. Warwick WI, Neal JM. Beyond spinal headache: prophylaxis and treatment of low-pressure headache syndromes. Reg Anesth Pain Med 2007;32:455-61

13. Diaz JH. Treatment outcomes in spontaneous intracranial hypotension: do epidural blood patches stop the leaks? Pain Pract 2004;4:295-302

14. Nowak DA, Takano B, Topka H. Spontaneous cerebrospinal fluid hypovolaemia: a therapeutic dilemma? Eur J Neurol 2006;13:1128-38

15. Hannerz J, Dahlgren G, Irestedt L, et al. Treatment of idiopathic intracranial hypotension: cervicothoracic and lumbar blood patch and peroral steroid treatment. Headache 2006;46:508-11

16. Bai J, Yokoyama K, Kinuya S, et al. Radionuclide cisternography in intracranial hypotension syndrome. Ann Nucl Med 2002;16:75-78

17. Schievink WI, Reimer R, Folger WN. Surgical treatment of spontaneous intracranial hypotension associated with a spinal arachnoid diverticulum: case report. J Neurosurg 1994;80:736-39

18. Schievink WI, Meyer FB, Atkinson JL, et al. Spontaneous spinal cerebrospinal fluid leaks and intracranial hypotension. J Neurosurg 1996;84:598-605

19. Brierley JB. The penetration of particulate matter from the cerebrospinal fluid into the spinal ganglia, peripheral nerves, and perivascular spaces of the central nervous system. J Neurol Neurosurg Psychiatry 1950;13:203-15

20. Irani DN. Cerebrospinal Fluid in Clinical Practice. Philadelphia: SaundersElsevier; 2009

21. Headache Classification Subcommittee of the International Headache Society. The International Classification of Headache Disorders. 2nd ed. Cephalalgia 2004;24(suppl 1):9-160

22. Schievink WI. Spontaneous spinal cerebrospinal fluid leaks and intracranial hypotension. JAMA 2006;295:2286-96

23. Schievink WI, Maya MM, Louy C, et al. Diagnostic criteria for spontaneous spinal CSF leaks and intracranial hypotension. AJNR Am J Neuroradiol 2008;29:853-56

24. Schievink WI. Misdiagnosis of spontaneous intracranial hypotension. Arch Neurol 2003;60:1713-18

25. Mokri B, Atkinson JL, Dodick DW, et al. Absent pachymeningeal gadolinium enhancement on cranial MRI despite symptomatic CSF leak. Neurology 1999;53:402-04

26. Mokri B. Intracranial hypertension after treatment of spontaneous cerebrospinal fluid leaks. Mayo Clin Proc 2002;77:1241-46

27. Medina JH, Abrams K, Falcone S, et al. Spinal imaging findings in spontaneous intracranial hypotension. AJR Am J Roentgenol 2010;195:459-64

28. Yousry I, Forderreuther S, Moriggl B, et al. Cervical MR imaging in postura headache: MR signs and pathophysiological implications. AJNR Am J Neuroradiol 2001;22:1239-50

29. Raymackers JM, Duprez T, Jeanjean A, et al. Discrepant time course of cranial and spinal subdural collections in a case of SIH treated by EBP. Eur Radiol 2001;11:2310-13 